\title{
LISTA RECENZENTÓW \\ „RUCHU PRAWNICZEGO, EKONOMICZNEGO I SOCJOLOGICZNEGO” \\ ZA ROK 2016
}

\author{
Teresa Bednarczyk \\ Barbara Będowska-Sójka \\ Stanisław Biernat \\ Wojciech Dajczak \\ Małgorzata Doman \\ Rafał Drozdowski \\ Aleksandra Duliniec \\ Maciej Duszczyk \\ Magdalena Fedorowicz \\ Beata Z. Filipiak \\ Kaja Gadowska \\ Anna Gerecka-Żołyńska \\ Włodzimierz Głodowski \\ Justyna Goździewicz-Biechońska \\ Andrzej Graczyk \\ Paweł Grzegorczyk \\ Janina Harasim \\ Zbigniew Janku \\ Elżbieta Jantoń-Drozdowska \\ Zdzisław Kędzia \\ Marian Kępiński \\ Krzysztof Knoppek \\ Hubert Kołecki \\ Marzena Kordela \\ Irena Lipowicz \\ Wojciech Łączkowski \\ Ewa Małuszyńska \\ Andrzej Matysiak \\ Tomasz Miziołek
}

Jacek Napierała

Tomasz Nieborak

Monika Oliwa-Ciesielska

Marcin Orlicki

Katarzyna Perez

Witold Płowiec

Łukasz Pohl

Jakub Pokrzywniak

Zbigniew Polański

Bożena Popowska

Wiesława Przybylska-Kapuścińska

Teresa Rabska

Marek Ratajczak

Mikołaj Rylski

Sebastian Samol

Michał Skapski

Tadeusz Skoczny

Andrzej Skoczylas

Marek Smolak

Tomasz Sójka

Tadeusz Stryjakiewicz

Hanna Suchocka

Jan Szambelańczyk

Piotr Szczepankowski

Marek Szczepański

Anna Szelagowska

Marek Szewczyk

Zbigniew Woźniak

Sławomira Wronkowska 